\title{
Electrokinetic Response of Double Layers
}

\author{
C.-Y. D. Lu ( 陸駿逸) \\ Department of Chemistry, National Taiwan University, Taipei 106, Taiwan, R.O.C.
}

\begin{abstract}
Based on electrokinetic equations, we calculate the dynamic response of the thin double layer of a charged surface. The double layer response acts as effective boundary conditions for the ion concentrations, flow field, and potential outside the double layer. The results can be used to further calculate the electrophoresis or the dielectric spectrum of the deformable charged suspensions.
\end{abstract}

Keywords: Electrokinetic effects; Zeta potential; Dielectrics; Electrophoresis.

\section{INTRODUCTION}

The effects of electric field on biological tissues such as cell suspensions have been studied from many disciplines. Electrophysiology, ${ }^{1}$ electrophoresis, and dielectric spectroscopy $^{1-2}$ are just a few well-known examples among a variety of interests. Learning from the well-studied colloid electrokinetic effects of the charged sphere suspension, one expects that the dynamics of electric potential, flow field, and ionic concentration will all be essential in providing a complete description of the response to the electric field. Biological systems have one more complication: the suspension has a complicated and deformable shape, which also evolves dynamically in response to the applied field. To develop an electrokinetic theory for biological systems is obviously a difficult task.

For a long time, colloid scientists have realized that the double layer region (near field) and the bulk fluid (far field), which excludes the double layer region, require different analyses. ${ }^{3-8}$ One has to first solve the far field and the near field separately; the two solutions are then matched at the edge of the double layer. Some implementations of the scheme either make use of the specific spherical geometry in an intrinsic way, ${ }^{4-5,7-8}$ and therefore are not adaptable to suspensions of other shapes.

For a suspension of an arbitrary shape, if the double layer is thin compared to the radius of curvature of the suspension, the generic near field can be analyzed as a planar problem. The generic near field, together with the matching conditions, acts as the effective boundary condition of the far field..$^{3-4,6,8,11}$ Such thin double layer theory is particularly use- ful for non-spherical suspensions. So far the thin double layer theories either focus on the relative high frequencies where the salt concentration is not perturbed, ${ }^{10}$ or are restricted to the steady state situation. ${ }^{7}$

Many theories are restricted to the high surface charge situation, where the double layer has strong effects (e.g. on the dielectric response). However, the analysis relies heavily on the assumption that the surface is charged. It is therefore difficult to make close comparisons with an uncharged reference system. The existing low surface charge theories are unfortunately restricted to the spherical geometry ${ }^{4-5,7-9}$ and can not easily be used for general suspension shapes.

In this paper we aim at a minimum thin double layer theory, which includes the full frequency spectrum below the Maxwell-Wagner relaxation rate with no restriction on the surface charge. We therefore focus on a simple situation where: (i) the double layer is thin, compared to the radius of curvature of the suspension; (ii) only the $z: z$ electrolyte is considered; and (iii) the counterion and the coion have the same mobility $\mu$. The assumption (i) is usually satisfied in the physiological condition where the salt concentration is high, e.g. for 0.001 molar $\mathrm{NaCl}$ solution, the Debye length is about $100 \AA$, which is much smaller than the typical cell size. Restrictions (ii) and (iii) greatly simplify the problem (see below). Nevertheless, the system still retains most of the qualitative features of the general electrokinetic effects, e.g. the full dielectric response spectrum. Developed along the line of the thin double layer theory, ${ }^{3-4,6,8,11}$ we obtain a complete set of the effective boundary conditions for the far field. In particular, the momentum discontinuity across the double layer is included, which can be used to calculate the cell deforma- 
tion. Also the high frequency Maxwell-Wagner theory and the low frequency salt polarization effect are included as special cases. With the help of the simplifications (ii) and (iii), the full range of the $\zeta$ potential is treated in a unified way. Therefore, uncharged, slightly charged, or strongly charged suspensions obey the same equations. The general solution scheme of the far field will not be discussed; the variety of the biological systems will eventually need a powerful numerical method to calculate the far field, which is outside our scope here.

\section{THEORY}

\section{Uncharged interface}

We will quickly review the boundary conditions required for a neutral interface so that the charged version given below has a clearer correspondence. Also, it is easier to count the number of boundary conditions required if the uncharged version is given first. They are velocity continuity, stress continuity, potential continuity, tangential electric field continuity, and normal electric displacement continuity. The bulk fluid obeys the (neutral) Navier-Stokes equation; inside the interface, other momentum equations needs to be supplied (e.g. elastic solid); the electric potential obeys the Laplace equation on the both sides of the interface.

\section{Electrokinetic equations}

The minimal description of the solution will involve the electric potential $\Psi$, the concentrations of the counterion $C_{+}$ and the coions $C_{-}$, and the flow field $\mathbf{v}$. They obey the Poisson equation, Smoluchowski equation, and the Navier-Stokes equation, respectively.

$$
\begin{aligned}
& \nabla^{2} \Psi=-q\left(\mathrm{C}_{+}-\mathrm{C}_{-}\right) / \varepsilon \\
& \partial_{t} \mathrm{C}_{ \pm}=\nabla \cdot\left(\mu T \nabla C_{ \pm} \pm q \mu C_{ \pm} \nabla \Psi-C_{ \pm} \mathbf{v}\right) \\
& \rho_{m}\left(\partial_{t}+\mathbf{v} \cdot \nabla\right) \mathbf{v}=-\nabla P-q\left(C_{+}-C_{-}\right) \nabla \Psi+\eta \nabla^{2} \mathbf{v}
\end{aligned}
$$

where the flow field is incompressible $\nabla \cdot \mathbf{v}=0, q$ is the counterion charge, $\mu$ the ion mobility, $T$ the temperature in energy unit, $\rho_{m}$ the mass density, $\eta$ the solvent viscosity, and $\varepsilon$ the dielectric constant of the solvent.

At the boundary of the suspensions, the velocity obeys the no slip boundary conditions. The stress is continuous. We assume that the ions can not penetrate into the suspensions. The tangential electric field is continuous. The normal electric displacement has a discontinuity. The magnitude of the discontinuity is determined by the surface charge density, which is assumed fixed here.

Based on the above complete electrokinetic equations, one can work out the far field equations valid outside the double layer (and the suspensions), and the near field solution within the double layer. Below we first review the far field equations, then sketch the near field solutions and give the effective far field boundary conditions.

\section{The far field equations}

Instead of $C_{+}$and $C_{-}$, one can use salt concentration perturbation $c \equiv\left(C_{+}+C_{-}\right) / 2-C_{0}$ and charge density $\rho=q\left(C_{+}-\right.$ $C_{-}$) to diagonalize partially eq. (2) where $C_{0}$ is the equilibrium salt concentration. The salt perturbation obeys the diffusion equation

$$
\left(\partial_{t}+\mathbf{v} \cdot \nabla\right) c=D \nabla^{2} c
$$

The diffusivity is $D=T \mu$. The charge density obeys

$$
\left(\partial_{t}+\mathbf{v} \cdot \nabla\right) \rho=D\left(\nabla^{2}-\kappa^{2}\right) \rho
$$

where the Poisson equation is used. The Debye length $\kappa^{-1}$ is defined by $\kappa^{2}=2 q^{2} C_{0} / \varepsilon T$. One can see that any charge excitation will not persist a few Debye lengths into the bulk; therefore, one can set $\rho=0$ in the bulk. The potential perturbation $\psi \equiv \Psi-\Psi_{0}$ therefore obeys (in the bulk) the Laplace equation

$$
\nabla^{2} \psi=0
$$

The fluid outside the double layer is also decoupled from the ion and potential

$$
\rho_{m}\left(\partial_{t}+\mathbf{v} \cdot \nabla\right) \mathbf{v}=-\nabla P+\eta \nabla^{2} \mathbf{v}
$$

The diffusion, Laplace and the (neutral) Navier-Stokes equations govern the salt concentration perturbation, the potential perturbation, and the fluid velocity exterior to the double layers. They coupled through the boundary conditions, which relate quantities on a mathematical surface adjacent, but exterior to the double layers. ${ }^{3-4,6,8}$ Below we first examine the double layer structure, then discuss our main goal, the boundary conditions.

\section{Quasistatic double layer}

From eq. (5), the charge will reach local equilibrium at the rate $\omega_{M W} \equiv K_{B} / \varepsilon=D \kappa^{2}$ where the bulk conductance is $K_{B}=$ 
$2 q^{2} \mu C_{0}$. The fluid inertia effect will relax at a rate $\omega_{S}=$ $\left(\eta / \rho_{m}\right) \kappa^{2}$ at the double layer scale. Given the ion diffusivity $10^{-9} \mathrm{~m}^{2} \mathrm{sec}^{-1}$ and the kinematics viscosity $\eta / \rho_{m}$ of water as $10^{-6} \mathrm{~m}^{2} \mathrm{sec}^{-1}$, a Debye length of $100 \AA$ gives very high $\omega_{M W}=$ $10 \mathrm{MHz}$ and $\omega_{S}=10 \mathrm{GHz}$ upper limit. We are interested mainly in large scale dynamics, which is far slower than $\omega_{M W}$ and $\omega_{s}$; therefore, we will assume the double layer is at local equilibrium.

The ion concentrations inside the double layer are $C_{ \pm}=$ $\left(C_{0}+\mathrm{c}_{\Lambda}\right) \exp \left(\overline{+} q\left(\Psi_{0}+\psi-\psi_{\Lambda}\right) / T\right)$ where the subscript $\Lambda$ means to evaluate the function at the mathematical surface $z$ $=\Lambda$, which is a few times the Debye length away from the interface (e.g. one may choose $\kappa^{-1}<\Lambda<10 \kappa^{-1}$ ).

We only consider the situation where the fluid can not permeate into the interface. The flow equation along the layer normal direction becomes the static condition $-\nabla_{z} P-\rho \nabla_{z} \Psi=$ 0 where $z$ is the coordinate along the surface normal direction. In the equilibrium $\rho=-2 C_{0} q \sinh (q \Psi / T)$, the pressure distribution is $P_{0}=2 T C_{0}(\cosh (q \Psi / T)-1)$. For a quasistatic double layer, one has

$$
P=P_{0}+2 T(\cosh (q \Psi / T)-1) c_{\Lambda}-\rho_{0}\left(\Psi-\Psi_{0}-\psi_{\Lambda}\right)
$$

inside the double layer.

Using the pressure given above, the tangential velocity (relative to the surface) in the double layer is obtained by integrating twice from $\eta \nabla_{z}^{2} \mathbf{v}_{\perp}=-\nabla_{\perp} P-\rho \nabla_{\perp} \Psi$ to give

$$
\mathbf{v}_{\perp}=\frac{\varepsilon\left(\zeta-\Psi_{0}\right)}{\eta} \nabla_{\perp} \Psi_{\Lambda}+\frac{8 T}{\eta \kappa^{2}} \ln \frac{\cosh q \Psi_{0} / 4 T}{\cosh q \zeta / 4 T} \nabla_{\perp} c_{\Lambda}
$$

where $\nabla_{\perp}$ denotes the in plane gradient.

\section{Velocity boundary conditions}

The velocity normal to the surface is continuous

$$
\left[\mathbf{v}_{z}\right]_{z=0}^{z=\Lambda}
$$

For the far field, which varies at a much larger length scale compared to $\Lambda$, the limit $\Lambda \rightarrow 0$ can be taken as a good approximation. Given the flow field, we obtain the tangential velocity boundary condition. The discontinuity of the tangential velocity is

$$
\left[\mathbf{v}_{\perp}\right]_{0}^{\Lambda}=\frac{\varepsilon \zeta}{\eta}\left(\nabla_{\perp} \psi_{\Lambda}-\frac{4 T}{q \zeta} \ln \left[\cosh \left(\frac{q \zeta}{4 T}\right)\right] \nabla_{\perp} \frac{T c_{\Lambda}}{q C_{0}}\right)
$$

Note that, on the right hand side, the limit $\Lambda \rightarrow \infty$ is taken, which is not physically inconsistent with the limit
$\Lambda \rightarrow 0$ on the left hand side, because we have considered the far field varies at a much larger length scale, say $L$, compared to the Debye length. The different limits are both reasonable approximations when $L>>\wedge>\kappa^{-1}$. The present calculation is basically the leading order result of the formal matched asymptotic analysis. To improve the result to include the finite ratio of $\Lambda / L$ and $1 /(\kappa \Lambda)$ will require the asymptotic analysis to be matched up to the next order in $1 /(\kappa L)$, which is beyond the scope of the present work. In that case one will encounter the higher order normal derivatives to enter into the boundary conditions, which extract the length scale $L$ from the yet to be determined far field solutions.

\section{Stress boundary conditions}

The shear stress $\Pi_{z \perp}(\perp=x$ or $y)$ has discontinuity across the double layer

$$
\left[\Pi_{z \perp}\right]_{0}^{\Lambda}=\Gamma\left(q \nabla_{\perp} \psi_{\Lambda}-\tanh \left(\frac{q \zeta}{4 T}\right) \nabla_{\perp} \frac{T c_{\Lambda}}{C_{0}}\right)
$$

where the $O(\Lambda)$ contributions are neglected. The surface excess ion density $\Gamma$ will be given below. The normal stress discontinuity follows the Laplace formula as

$$
\left[\Pi_{z z}\right]_{0}^{\Lambda}=2 \tau H
$$

where $H$ is the mean of the two principle curvatures. The surface tension of the diffuse layer $\tau$ is given by

$$
\tau \equiv \int_{0}^{\Lambda}\left(\Pi_{\perp}-\Pi_{z z}\right) d z=-2 T \Gamma
$$

Note that the surface tension is negative. This is consistent with the Gibbs' adsorption equation that the positive adsorption on the surface lowers the surface tension.

\section{Surface excess}

Define the surface excess ionic concentration $\Gamma$ and surface excess charge $\Sigma$ as

$$
\begin{aligned}
& \Gamma \equiv \int_{0}^{\Lambda}\left(C_{+}+C_{-}-2 C_{0}-2 c_{\Lambda}\right) d z=\frac{8 C_{0}}{\kappa} \sinh ^{2} \frac{q \zeta}{4 T} \\
& \Sigma \equiv q \int_{0}^{\Lambda}\left(C_{+}-C_{-}\right) d z=-q \frac{4 C_{0}}{\kappa} \sinh \frac{q \zeta}{2 T}
\end{aligned}
$$

Note that the quasistatic double layer is fully characterized by the salt concentration nearby and the $\zeta$ potential.

We choose to analyze the surface excess because it is not sensitive to the exact value of $\Lambda$, so the convenient limit $\Lambda \rightarrow \infty$ can be taken in the integral to reduce a free parameter. 
As will be discussed below, the surface excess obeys the (mass) conservation law, which can be derived from eqs. (1)-(3). Note that instead of the individual ion conservations, we choose to analyze the surface charge conservation (and the somewhat arbitrary ion concentration conservation) so that the connection with the MW theory is clearer.

Expressing the perturbation $\gamma$ and $\sigma$ (of $\Gamma$ and $\Sigma$, respectively) in terms of the salt perturbation $c_{\Lambda}$ and the $\zeta$ potential perturbation $\delta \zeta \equiv[\psi]_{0}^{\Lambda}=\psi(x, y, 0)-\psi(x, y, z=\Lambda)$, we have

$$
\begin{aligned}
& \gamma=\frac{\Gamma}{2 C_{0}} c_{\Lambda}-\frac{q \Gamma}{2 T} \operatorname{coth}\left(\frac{q \zeta}{4 T}\right)[\psi]_{0}^{\Lambda} \\
& \sigma=\frac{\Sigma}{2 C_{0}} c_{\Lambda}-\frac{q \Sigma}{2 T} \operatorname{coth}\left(\frac{q \zeta}{4 T}\right)[\psi]_{0}^{\Lambda}
\end{aligned}
$$

The time derivatives are

$$
\begin{aligned}
& \partial_{t} \gamma=\frac{\Gamma}{2 C_{0}} \partial_{t} c_{\Lambda}-\frac{q \Gamma}{2 T} \operatorname{coth}\left(\frac{q \zeta}{4 T}\right) \partial_{t}[\psi]_{0}^{\Lambda} \\
& \partial_{t} \sigma=\frac{\Sigma}{2 C_{0}} \partial_{t} c_{\Lambda}-\frac{q \Sigma}{2 T} \operatorname{coth}\left(\frac{q \zeta}{4 T}\right) \partial_{t}[\psi]_{0}^{\Lambda}
\end{aligned}
$$

which will be used below.

\section{Surface excess conservation}

The conservation law of the excess ion density can be obtained by calculating the normal and the tangential flux contributions of a small piece of the double layer. Using essentially the same method as Fixman, ${ }^{4}$ we obtain

$$
\partial_{t} \gamma=2 D\left(\nabla_{z} c\right)_{\Lambda}+\left(K_{\gamma \psi} \nabla_{\perp}^{2} q \psi+K_{\gamma c} \nabla_{\perp}^{2} \frac{T c}{C_{0}}\right)_{\Lambda}
$$

The conservation of the excess charge density is

$$
\partial_{t} \sigma=K_{B}\left(\nabla_{z} \psi\right)_{\Lambda}+\left(K_{\sigma \psi} \nabla_{\perp}^{2} q \psi+K_{\sigma c} \nabla_{\perp}^{2} \frac{T c}{C_{0}}\right)_{\Lambda}
$$

where the coefficients $K^{\prime}$ s are

$$
\begin{aligned}
& K_{\gamma \psi}=\frac{\Sigma}{q}\left(\mu+\frac{4 C_{0}}{\eta \kappa^{2}}\right)+\frac{8 C_{0}^{2} q \zeta}{\eta \kappa^{3} T} \\
& K_{\gamma c}=\Gamma\left(\mu+\frac{4 C_{0}}{\eta \kappa^{2}}\right)-\frac{32 C_{0}^{2}}{\eta \kappa^{3}} \ln \left(\cosh ^{2} \frac{q \zeta}{4 T}\right) \\
& K_{\sigma \psi}=q^{2} \Gamma\left(\mu+\frac{4 C_{0}}{\eta \kappa^{2}}\right) \\
& K_{\sigma c}=q^{2} K_{\gamma \psi}
\end{aligned}
$$

The terms with $\eta$ are coming from the convection flux which can be calculated using the flow field inside the double layer eq. (9).

Different from the salt polarization theories, ${ }^{3-4,8}$ we do not neglect the time derivative quantities, which are essential in order to recover the Maxwell-Wagner theory of the interfacial polarization. ${ }^{1-2,10}$

\section{The electric field}

Followed the Gauss law, the perturbed potential has a discontinuity across the double layer

$$
-\left[\varepsilon \nabla_{z} \psi\right]_{0}^{\Lambda}=\sigma=\frac{\Sigma}{2 C_{0}} c_{\Lambda}-\frac{q \Sigma}{2 T} \operatorname{coth}\left(\frac{q \zeta}{4 T}\right)[\psi]_{0}^{\Lambda}
$$

\section{SUMMARY}

In summary, the full properties of the double layer are characterized in the boundary conditions (10) (11) (12) (13) (18) (19) (20) (21) (23) for the various discontinuities of the perturbation quantities, which obey eqs. (4) (6) (7) in the bulk solution. This can be used as a starting point to calculate, e.g., the full electrophoresis velocity of the cells.

Note that the far field flow velocity is important for electrophoresis, but it is much less important for linear dielectric response, ${ }^{9}$ because the far field only convects salt fluctuation, producing the effect of quadratic order in the applied field strength. Therefore for dielectric response, it is sufficient to solve a subset of the equations for potential and the salt concentration only.

\section{ACKNOWLEDGEMENTS}

The author would like to thank the National Science Council of the Republic of China, Taiwan for financially supporting this research under Contract No. NSC91-2119-M027-CC3.

Received February 16, 2005.

\section{REFERENCES}

1. Takashima, S. Electrical Properties of Biopolymers and Membranes; Adam Hilger: Bristol, 1989. 
2. Foster, K. R.; Schwan, H. P. In Handbook of Biological Effects of Electromagnetic Radiation; Polk, C. and Postow, E., Eds., CRC press, Boca Raton, Fla., 2 ${ }^{\text {nd }}$. edn. 1996.

3. Dukhin, S. S.; Shilov, V. N. Dielectric Phenomena and the Double Layer in Disperse Systems and Polyelectrolytes; Wiley: New York, 1974.

4. Fixman, M. J. Chem. Phys. 1980, 72, 5177; 1983, 78, 1483.

5. De Lacey, E. H. B.; White, L. R. J. Chem. Soc. Faraday Trans. 2 1981, 77, 2001.
6. O’Brien, R. W. J. Colloid Interface Sci. 1983, 92, 204.

7. O'Brien, R. W. Adv. Colloid Interface Sci. 1982, 16, 281.

8. Hinch, E. J.; Sherwood, J. D.; Chew, W. C.; Sen, P. N. J. Chem. Soc. Faraday Trans. 2 1984, 80, 535.

9. Mangelsdorf, C. S.; White, L. R. J. Colloid Interface Sci. 1993, 160, 275.

10. O’Brien, R. W. J. Colloid Interface Sci. 1986, 113, 81.

11. Lu, C.-Y. D. Europhys. Lett. 1996, 36, 215. 